\title{
A PRIMARY QUANTUM MODEL OF TELEPATHY
}

\author{
Gao Shan \\ The Scientists Work Team of Electro-Magnetic Wave Velocity, \\ Chinese Institute of Electronics and Institute of Quantum Physics, Beijing
}

\begin{abstract}
The physical nature of psi phenomena such as telepathy is an important problem in present science of consciousness. Scientists have basically confirmed the existence of telepathy phenomena through many strict experiments. Then can modern science (e.g. quantum theory) provide a scientific explanation for telepathy phenomena? In this paper, we will seek the possible quantum nature of telepathy from both theoretical and experimental aspects, and will present a primary quantum model of telepathy process. It is well known that even though present quantum theory permits the existence of quantum nonlocality, it does forbid the realization of nonlocal communication or quantum superluminal communication (QSC). However, the usual no-go theorems don't consider the possible active role of consciousness during quantum measurement process. In a recent paper (see Found. Phys. Lett, 17(2), 167-182), it has been demonstrated that a proper combination of quantum process and conscious perception will permit the distinguishability of nonorthogonal quantum states, and further result in the realization of QSC or nonlocal communication. This is called the QSC principle. On the basis of the QSC principle, we propose a primary theoretical model of telepathy process. According to the model, the telepathy process mainly includes three phases. The first phase is to form the quantum entanglement state of brains, the second phase is to hold the entanglement state of brains, and the third phase is to collapse the entanglement state of brains. When the entanglement state of brains is collapsed by a certain measurement on one of the subjects, the brain states of both subjects turn to be definite states from entanglement state, and the other subject will perceive the change at a distance according to the QSC principle. When in the entanglement state or superposition state, no definite perception relating to the state exists, whereas when the superposition state collapses into a definite state, a definite perception relating to the collapse state appears. Then the telepathy between the subjects may appear. It should be stressed that, even though the above quantum model may in principle provide a primary scientific explanation of telepathy phenomena, there are still some left technical problems such as the expression of high-level telepathy information etc. Lastly, in order to test the QSC principle and the above quantum model of telepathy, some feasible schemes of quantum perception experiments and perception entanglement experiments are further proposed on the basis of present technology. We urge that such quantum perception experiments need to be conducted as soon as possible. If the experiment results are positive, they will have far-reaching influence on the present science of consciousness and psi research, and will help to bridge the gap between the parapsychology and present science.
\end{abstract}

\section{INTRODUCTION}

The physical nature of psi phenomena such as telepathy is an important problem in present science of consciousness. The existence of telepathy phenomena has been basically confirmed through many strict experiments (Duane \& Behrendt, 1965; Targ \& Puthoff, 1974; Puthoff \& Targ, 1976; Radin \& Nelson, 1989; Grinberg-Zylberbaum et al, 1994; Bierman \& Radin, 1997; Gao, 2000; Wackermann et al, 2003). Then can modern science (e.g. quantum theory) provide a scientific explanation for telepathy phenomena? In this paper, we will seek the possible quantum nature of telepathy from both theoretical and experimental aspects, and will present a primary quantum model of telepathy phenomena. It will be shown that, according to the principle of quantum superluminal communication (QSC) (Gao, 2000; Gao, 2003; Gao, 2004), quantum theory can in principle provide a scientific explanation of telepathy phenomena when considering the role of consciousness in quantum process, and some experiments may have indicated the validity of this explanation. Lastly, we will propose a series of feasible experiment schemes to test the quantum model of telepathy. 


\section{A ROLE OF CONSCIOUSNESS IN QUANTUM MEASUREMENT}

We will first analyze the role of consciousness in physical measurement process. As we know, physical measurement generally consists of two processes: (1). the physical interaction between the observed object and measuring device; (2). the psycho-physical interaction between the measuring device and observer. In some special situations, measurement may be the direct interaction between the observed object and observer.

Even though what physics studies is the insensible object or matter, the consciousness of the observer must take part in the last phase of measurement. The observer is introspectively aware of his perception about the measurement results. The conscious function is used to end the infinite chains of measurement. This is the main role of consciousness different from that of usual measuring device in the measurement process. But this difference doesn't result in the physically testable different displays for classical measurement process. In classical theory, the influence of the measuring device or observer to the observed object can be omitted in principle during measurement process, and the psycho-physical interaction between the observer and measuring device does not influence the reading of the pointer of the measuring device either. Thus classical measurement is only one kind of plain one-to-one mapping from the state of the observed object to the pointer state of the measuring device and further to the mental state of the observer, or direct one-to-one mapping from the state of the observed object to the mental state of the observer from a physical point of views. In short, the consciousness of the observer possesses no physically testable different functions from physical measuring device in classical theory.

However, the measurement process is no longer plain in quantum theory. The influence of the measuring device to the observed object can't be omitted in principle during quantum measurement owing to the existence of quantum superposition. It is just this influence that generates the definite measurement result to some extent. Since the measuring device has generated one definite measurement result, the psycho-physical interaction between the observer and measuring device is still one kind of plain one-to-one mapping, and this process is the same as that in classical situation. But when the observed object and observer directly interact, the existence of quantum superposition will introduce new element to the psychophysical interaction between the observer and measured object. The interaction will result in the appearance of the observer with consciousness in quantum superposition state. Then whether or not does the consciousness of the observer in quantum superposition state have some physically testable different displays from physical measuring device? We will try to give the answer.

In order to further analyze the possible role of consciousness during quantum measurement, we need a complete theory describing the quantum measurement process. As we know, present quantum theory hasn't provided a complete description of the evolution of wave function during measurement yet, and the projection postulate is just a makeshift. Revised quantum dynamics (Ghirardi et al, 1986; Pearle, 1989; Diosi, 1989; Ghirardi et al, 1990; Penrose, 1996; Gao, 2000; Gao, 2001; Gao, 2003) and many-worlds theory (Everett, 1957; Dewitt et al, 1973; Deutsch, 1985) are two main alternatives to a complete quantum theory. Here we mainly discuss the measurement process in the framework of revised quantum dynamics, and the conclusion will be also valid in the many-worlds theory. At the present time, even if the last complete theory has not been found, but one thing is certain, i.e. the collapse process of wave function is one kind of dynamical process, and it will take a finite time interval to finish. Our analysis will only rely on this common character of the complete quantum theory.

As we know, the nonorthogonal quantum states or nonorthogonal states such as $\psi_{1}$ and $\psi_{1}+\psi_{2}$ can't be distinguished in present quantum theory. What's more, the usual measurement using physical measuring device can't distinguish the nonorthogonal states either in the framework of revised quantum dynamics. But when the physical measuring device is replaced by a conscious being and considering the influence of consciousness, it has been shown that the nonorthogonal states can be distinguished under some condition using the consciousness function (Gao, 2000; Gao, 2003; Gao, 2004). The observer with consciousness may obtain more information about the intermediate process before the collapse of wave function finishes, and 
the added information can help him distinguish the nonorthogonal states. This is the special role of consciousness different from that of physical measuring device during quantum measurement process. Here we will introduce the main ideas.

Let the states to be distinguished be the nonorthogonal states $\psi_{1}$ and $\psi_{1}+\psi_{2}$, where $\psi_{1}$ and $\psi_{2}$ can trigger the definite perception states $\chi_{1}$ and $\chi_{2}$ of the observer, and the initial perception state of the observer be $\chi_{0}$. After interaction the corresponding entangled state of the whole system is respectively $\psi_{1} \chi_{1}$ and $\psi_{1} \chi_{1}+\psi_{2} \chi_{2}$. We assume that the observer satisfies the "QSC condition", i.e., his perception time for the definite state $\psi_{1} \chi_{1}$, which is denoted by $t_{P}$, is shorter than the dynamical collapse time of the superposition state $\psi_{1} \chi_{1}+\psi_{2} \chi_{2}$, which is denoted by $t_{C}$, and that the time difference $\Delta t=t_{C}-t_{P}$ is large enough for him to identify. The observer can perceive the input definite state $\psi_{1}$ after the perception time $t_{P}$, whereas for the input superposition state $\psi_{1}+\psi_{2}$, only after the collapse time $t_{C}$ can the observer perceive the collapse state $\psi_{1}$ or $\psi_{2}$. Before the collapse time $t_{C}$ the observer in superposition state $\psi_{1} \chi_{1}+\psi_{2} \chi_{2}$ has no definite perception related to the definite state $\psi_{1}$ or $\psi_{2}$; After the collapse time $t_{C}$, the state of the measured system collapses to a definite state $\psi_{1}$ or $\psi_{2}$, and the observer has a definite perception for the collapse state $\psi_{1}$ or $\psi_{2}$. Since the observer can be conscious of the time difference between $t_{P}$ and $t_{C}$, he can distinguish the nonorthogonal states $\psi_{1}$ and $\psi_{1}+\psi_{2}$. It should be stressed that, since the collapse time of a single superposition state is an essentially stochastic variable, which average value is $t_{C}$, the "QSC condition" can be in principle satisfied in some collapse processes. For these stochastic collapse processes, the collapse time of the single superposition state is much longer than the (average) collapse time $t_{C}$ and the perception time $t_{P}$.

\section{A NONLOCAL COMMUNICATION METHOD}

It is well known that even though present quantum theory permits the existence of quantum nonlocality (Einstein et al, 1935; Bell, 1964; Aspect, 1982), it doesn't permit the realization of quantum superluminal communication (QSC) (Eberhard, 1978; Ghirardi, 1980). However, such demonstrations didn't consider the possible active role of consciousness during quantum measurement process. As we have demonstrated above, a proper combination of quantum process and conscious perception will permit the distinguishability of nonorthogonal states. Once the nonorthogonal states can be distinguished, we can directly realize QSC or nonlocal communication. This is called the QSC principle. Here we present a typical method to realize nonlocal communication.

According to the above analysis, we can design a device to distinguish the nonorthogonal states. We call it NSDD (Nonorthogonal States Distinguishing Device). The design rules are as follows, i.e., when the input state is a definite state, the output of NSDD is ' 1 ', whereas when the input state is a superposition of definite states, the output of NSDD is ' $O$ '. In the following, we will briefly introduce how to achieve QSC using NSDD. 


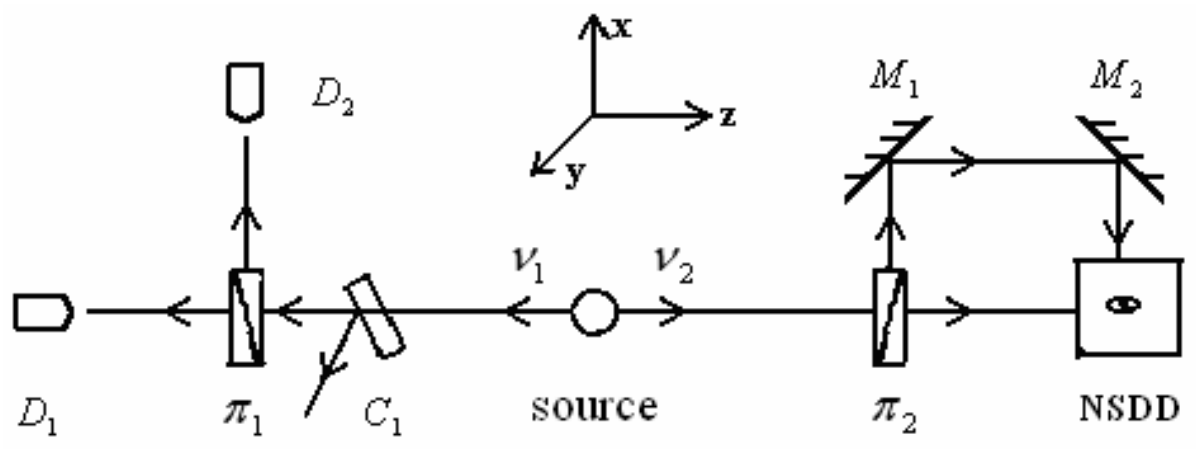

Fig. 1: A scheme of QSC

We use the EPR polarization correlation pairs of photons as the carriers of information. We encode the outgoing information by operating the polarizer, and decode the incoming information using NSDD. The experimental setting is shown in Fig 2. Pairs of photons, whose frequencies are $v_{1}$ and $v_{2}$, are emitted in the $-z$ direction and $+z$ direction from a source, are then analyzed by the two-channel polarizer $\pi_{1}$ and $\pi_{2}$ respectively. The optical switch $C_{1}$ in the left side can be controlled to determine whether or not the photon $v_{1}$ will pass to $\pi_{1}$. The transmission axes of the polarizers are both set in the direction $\mathrm{x}$. The twochannel polarizer $\pi_{1}$ and $\pi_{2}$ allows the polarization components of the photon both parallel to and perpendicular to the transmission axis of the polarizer to be passed. The photon passed and analyzed by the polarizer $\pi_{1}$ is detected by $D_{1}$ or $D_{2}$, and the photon analyzed by the two-channel polarizers $\pi_{2}$ is divided into two paths in space, and respectively input to NSDD from different directions.

We now explain how QSC can be achieved by means of the above setting. Let the sender operate the optical switch $C_{1}$, and have the receiver observe the output of NSDD. Suppose the communication rules are stated as follows. The encoding rule for the sender is that not measuring the photon represents sending the code ' 0 ', and measuring the photon represents sending the code ' 1 '. The decoding rule for the receiver is that the output of NSDD being ' $O$ ' represents having received the code ' $O$ ', and the output of NSDD being ' 1 ' represents having received the code ' 1 '. The communication process can be stated as follows. When the sender wants to send a code ' $O$ ', he controls the optical switch $C_{1}$ to let the photon $v_{1}$ move freely and not be analyzed by the polarizer $\pi_{1}$. Then the state of the photon $v_{2}$ is a superposition state after it passes the polarizer $\pi_{2}$, and the output of NSDD is ' 0 '. The receiver can decode the sent code as ' 0 '. When the sender wants to send a code ' 1 ', he controls the optical switch $C_{1}$ to allow the photon $v_{1}$ to be analyzed by the polarizer $\pi_{1}$ and detected by $D_{1}$ or $D_{2}$ before the photon $v_{2}$ arrives at NSDD. Then the state of the photon $v_{2}$ collapses into a definite state, and the output of NSDD is ' 1 '. The receiver can decode the sent code as ' 1 '. Thus the sender and receiver can achieve QSC using the above setting and communication rules.

\section{TELEPATHY AND ITS POSSIBLE QUANTUM EXPLANATION}

Even though some superphysical phenomena may be not real, telepathy does exist. Its usual display is that one can perceive the other's happening, say being sick or being injured etc, at a distance between the familiar people, say twins, relatives or friends. Many people have this kind of experience. At present, the telepathy phenomena have been basically confirmed by some strict experiments (Duane \& Behrendt, 1965; 
Targ \& Puthoff, 1974; Puthoff \& Targ, 1976; Radin \& Nelson, 1989; Grinberg-Zylberbaum et al, 1994; Bierman \& Radin, 1997; Gao, 2000; Wackermann et al, 2003), and are being studied by more scientists.

In the experiment conducted by Grinberg-Zylberbaum et al (Grinberg-Zylberbaum et al, 1994), pairs of subjects were first allowed to meditate together, and then put into two semisilent Faraday chambers $14.5 \mathrm{~m}$ apart. Their EEG activities are registered by two EEG machines. One subject of each pair was stimulated by 100 flashes at random intervals, and each photostimulation resulted in an evoked potential for the stimulated subject. It is observed that, when the stimulated subject showed distinct evoked potentials, the nonstimulated subject showed "transferred potentials" similar to the evoked potentials in the stimulated subject, at the same time, the subjects both felt their interaction had been successfully completed. In another experiment conducted by Wackermann et al (Wackermann et al, 2003), six channels electroencephalogram (EEG) were recorded simultaneously from pairs of separated human subjects in two acoustically and electromagnetically shielded rooms. Even though the "transferred potentials" is not found in the experiment, the correlations between brain electrical activities of two spatially separated human subjects are also observed using a more complex method of data analysis. Since the subjects were separated by the soundproof Faraday chambers in the above experiments, these experiments guarantee that neither sensory signals nor electromagnetic signals is the means of communication, and thus strictly confirms the existence of nonlocal correlations and nonlocal communication between human brains.

In the following, we will analyze the above telepathy experiments in terms of the above QSC principle. According to the QSC principle, the proper combination of quantum collapse and conscious perception will result in the realization of nonlocal communication. It will be shown that this may provide a possible explanation of the above telepathy experiments, and indicates that telepathy may result from the quantum process in brains.

We first argue that the "QSC condition" is satisfied in the above telepathy experiment as implied by the experiment results. The "QSC condition" is that the perception time of a conscious being for the definite state is shorter than the dynamical collapse time of the perceived quantum superposition state, and the time difference is large enough for the conscious being to identify. On the one hand, the quantum entanglement state between the subjects A and B in the experiment, which is formed by meditative interaction or other means, can hold for a long time until the experiment is completed, then there appears the observed correlations between brain electrical activities of the two subjects. This indicates that the dynamical collapse time of the quantum entanglement state is also very long, say several ten minutes. On the other hand, the perception time of the subjects for the definite state is generally of the orders of $500 \mathrm{~ms}$. Thus in the above experiments the collapse time of the quantum entanglement state or quantum superposition state is evidently much longer than the perception time of the subject for the definite state, and the time difference is also large enough for the subject to identify, i.e., the "QSC condition" is satisfied in the experiments.

It seems to be a well-known fact that the wet and warm brain doesn't support the quantum coherence (Tegmark, 2000). However, on the one hand, the "QSC condition" is related to the collapse time, not the decoherence time of wave function. Even though the decoherence time is very short due to environmental decoherence, the collapse time may be much longer (Hagan et al, 2002). Here we will also give an example. As we know, the number of neurons which can form a definite conscious perception is in the levels of $10^{4}$. In each neuron, the main difference of activation state and resting state lies in the motion of $10^{6} \mathrm{Na}^{+} \mathrm{s}$ passing through the membrane. Since the membrane potential is in the levels of $10^{-2} \mathrm{~V}$, the energy difference between activation state and resting state is approximately $10^{4} \mathrm{eV}$. According to one kind of revised quantum dynamical theory (Percival, 1994; Hughston, 1996; Fivel, 1997; Gao, 2000; Gao, 2003), the collapse time of the superposition of the activation state and resting state of one neuron is $\tau_{c} \approx \frac{\hbar E_{p}}{(\Delta E)^{2}} \approx\left(\frac{2.8 \mathrm{MeV}}{0.01 \mathrm{MeV}}\right)^{2} \approx 10^{5} s$, where $\hbar$ is Planck constant divided by $2 \pi, E_{p}$ is Planck energy, $\Delta E$ is the energy difference of the state. Thus the collapse time of the superposition of two different 
conscious perceptions is $\tau_{c} \approx\left(\frac{2.8 \mathrm{Mev}}{100 \mathrm{MeV}}\right)^{2} \approx 1 \mathrm{~ms}$, in which one conscious perception state contains $10^{4}$ neurons in the activation state, and the other conscious perception state contains $10^{4}$ neurons in the resting state. Since the collapse process is an essentially stochastic process, and the collapse time of a single superposition state is a stochastic variable, which average value $\tau_{c}$ is nearly $1 m s$, the "QSC condition" can be in principle satisfied in some collapse processes happening in the brains. For these stochastic collapse processes, the collapse time of the single superposition state is much longer than the average perception time $500 \mathrm{~ms}$. This may account for the experimental results of the above telepathy experiments.

Once the required "QSC condition" is satisfied, realizing QSC and explaining telepathy will be probable. According to the QSC principle, the subject satisfying the "QSC condition" will possess different perceptions for the definite state and the superposition of definite states. As revealed in the experiment, when the subject $A$ is not stimulated and the quantum entanglement state still holds, the subject $B$ will be in a superposition state, and he has no distinct feeling or distinct distributions of the brain electrical activities related to the state. Whereas when the subject $\mathrm{A}$ is stimulated and the quantum entanglement state collapses, the subject $\mathrm{B}$ will be in a definite state, and he does have a distinct feeling that their interaction has been successfully completed or distinct distributions of the brain electrical activities. Then QSC can be realized if we encode the different stimulating operations to subject $A$, and correspondingly decode the codes through the different feelings or EEG activities of subject B. This may naturally explain the telepathy phenomenon between the subjects.

\section{A QUANTUM THEORETICAL MODEL OF TELEPATHY PROCESS}

On the basis of the QSC principle and the above analyses, we will further present a primary theoretical model of telepathy process. In this model, the telepathy process includes three main phases.

Phase 1: Form the quantum entanglement state of brains

During this phase, the quantum states of the brains of the telepathy subjects are entangled. Here we give a possible way to entangle the quantum states of brains. Suppose two photons are in the entanglement state $\psi_{1} \varphi_{2}+\varphi_{1} \psi_{2}$, and they respectively enter the eyes of two subjects A and B whose initial states is respectively $\chi_{0}(A)$ and $\chi_{0}(B)$. Then after interaction the entanglement state of these two brains will be formed, which can be written as $\chi_{1}(A) \chi_{2}(B)+\chi_{2}(A) \chi_{1}(B)$. Here we assume that the photons are absorbed in the process. In the above experiments, this phase is achieved by the meditative interaction or other interactions between the subjects.

Phase 2: Hold the entanglement state of brains

The formed entanglement state of brains may hold for a long time in some places of brain under some special conditions. According to the QSC principle, the holding time should be much longer than the usual perception time of the subjects. It is argued that this condition may be satisfied in some places of the brain (Penrose, 1994; Hameroff \& Penrose, 1996; Hagan et al, 2002). In the above experiments, the entanglement state is hold by the subjects feeling each other's presence at a distance.

Phase 3: Collapse the entanglement state of brains

When the entanglement state of brains is collapsed by a certain measurement on one of the subjects, the brain states of both subjects turn to be definite states from entanglement state, and the other subject will perceive the change at a distance according to the QSC principle. Here the telepathy between the subjects appears. When in the entanglement state or superposition state such as $\chi_{1}(A) \chi_{2}(B)+\chi_{2}(A) \chi_{1}(B)$, no definite perception relating to the state exists, whereas when the superposition state collapses into a definite state $\chi_{1}(A) \chi_{2}(B)$ or $\chi_{2}(A) \chi_{1}(B)$, a definite perception relating to the collapse state can appear. In the above experiment, this phase is achieved by stimulating the subject A with flashes or visual patterns, and 
when the entanglement state is collapsed by the stimulation, the subjects will display distinct distributions of the brain electrical activities or even feel that their interaction has been successfully completed.

It should be stressed that, even though the above primary quantum model may in principle provide a scientific explanation of telepathy phenomena, there are still some left technical problems. One is to find the position in the brain where the holding time of a quantum superposition state can be much longer than the usual perception time, i.e., to test the existence of "QSC condition" in human brains. Another problem is to study how the brain generates the high-level telepathy information from the low-level one transmitted through the above QSC means. This closely relates to present neuroscience research. Undoubtedly, these unsolved problems need to be deeply studied in experiments. In the next section, we will suggest some experimental schemes that may help to solve the problems.

\section{SOME EXPERIMENTAL SCHEMES}

In order to test the existence of "QSC condition" in human brains, and confirm the above primary quantum model of telepathy, we propose the following experimental schemes.

\section{Control experiment}

Produce some photons with a certain frequency. Input them to the eyes of the subject. Test and record the conscious time of the subject through EEG (electroencephalograph) or his oral description.

\section{Quantum perception experiment I}

Produce the direction superposition state of the photons with the same frequency (e.g. as stated in section 2). Input one branch of the superposition state to the eyes of the subject, and let the other branch freely spread (not input to a measuring device). Test whether the subject perceives the photons during the normal conscious time.

\section{Quantum perception experiment II}

Produce the direction superposition state of the photons with the same frequency. Input both branches of the superposition state to the eyes of the subject. Test whether the subject perceives the photons during the normal conscious time.

\section{Perceptions entanglement experiment I}

Produce the direction superposition state of the photons with the same frequency. Input the branches of the superposition state to the eyes of two independent subjects respectively. Test whether the subjects perceive the photons during the normal conscious time. It is suggested that the subjects are unfamiliar with each other before the experiment, which can be further confirmed by the phase incoherence of their brain waves.

If the subjects can only perceive the photons after a time interval longer than their normal conscious time in any case of the above experiments, then we will have confirmed the existence of "QSC condition" in human brains. Besides, we suggest that the subjects in the above experiments should be composed of three independent groups at least. The subjects in the first group are in normal state, the subjects in the second group are in meditation state, and the subjects in the third group are in qigong state.

\section{Perceptions entanglement experiment II}

Produce the direction superposition state of the photons with the same frequency. Input the branches of the superposition state to the eyes of two independent and isolated subjects respectively. Then stimulate one of the subjects using flashes at random intervals. Record his evoked potentials and the corresponding brain 
electrical activities of the other subject. Test whether there exists statistical relevance between these brain electrical activities. At the same time, ask the subjects whether they have some kind of conscious perception relating to the stimulations. The existence of the correlation of the brain electrical activities or the direct perception will have confirmed the above primary quantum model, and it can be used to realize controllable human brain communication.

This experiment can be taken as the quantum version of the above telepathy experiments. It is further suggested that the experiment be conducted at much longer distance, e.g. at a distance longer than the bound distance $40 \mathrm{~km}$. The present experimental results have shown that the maximum time delay between the EEG response of the receiver and the evoked potentials of the sender is approximately $130 \mu \mathrm{s}$ (Grinberg-Zylberbaum et al, 1994; Wackermann et al, 2003). Considering the value of light speed, the bound distance excluding the influence of classical signals with light speed is approximately $40 \mathrm{~km}$. Here the possible classical signals with light speed can't be used to explain the statistical relevance between the potentials of the subjects. Thus we can strictly confirm that the possible human brain communication is one kind of superluminal and non-electromagnetic phenomena, and further confirm the proposed quantum model of telepathy.

\section{REFERENCES}

Aspect A., Dalibard J. \& Roger G. (1982). Experimental Test of Bell's Inequalities Using Time-Varying Analyzers. Phys.Rev.Lett 49, 1804.

Bell J.S. (1964). On the Einstein Podolsky Rosen Paradox. Physics 1, 195.

Bierman, D.J. \& Radin, D. I. (1997). Anomalous anticipatory response on randomized future conditions. Perceptual and Motor Skills 84, 689-690.

Deutsch, D. (1985). Quantum Theory as a Universal Physical Theory. Int. J. Theo. Phys. 24, 1-41.

DeWitt, B. S. \& Graham, N. ed. (1973). The Many-Worlds Interpretation of Quantum Mechanics. Princeton: Princeton University Press.

Diosi, L. (1989). Models for universal reduction of macroscopic quantum fluctuations. Phys. Rev. A, 40, 1165-1174.

Duane, D. \& Behrendt, T. (1965). Extrasensory electroencephalographic induction between identical twins. Science, $150,367$.

Eberhard P .H. (1978). Bell's theorem and the different concepts of locality. Nuovo Cimento B, 46, 392.

Einstein A., Podolsky B. \& Rosen N. (1935). Can quantum mechanical description of physical reality be considered complete? Phys. Rev. 47, 777-780.

Everett, H. (1957). "Relative state" formulation of quantum mechanics. Rev. Mod. Phys. 29, 454-462.

Fivel, D. I. (1997). An indication from the magnitude of CP violations that gravitation is a possible cause of wavefunction collapse, LANL e-print quant-ph/9710042.

Gao Shan (2000). Quantum Motion and Superluminal Communication. Beijing: Chinese BT Publishing House.

Gao Shan (2001). From quantum motion to classical motion-seeking the lost reality. Physics Essays, 14(1), 37-48.

Gao Shan (2003). Quantum. Beijing: Tsinghua University Press.

Gao Shan (2004). Quantum collapse, consciousness and superluminal communication. Found. Phys. Lett, 17(2), 167182.

Ghirardi G.C., Rimini A. \& Weber T. (1980). A general argument against superluminal transmission through the quantum mechanical measurement process. Letters Nuovo Cimento. 27, 293.

Ghirardi,G.C., Rimini, A. \& Weber, T. (1986). Unified dynamics for microscopic and macroscopic systems. Phys. Rev. $D, 34,470-491$. 
Ghirardi,G.C., Rimini, A. \& Weber, T. (1990). A Continuous-spontaneous-reduction model involving gravity. Phys. Rev. D, 42, 1057-1064.

Grinberg-Zylberbaum, J., Dalaflor, D., Attie, L. \& Goswami, A. (1994). The Einstein- Podolsky-Rosen paradox in the brain: The transferred potential. Physics Essays, 7, 422.

Hagan S., Hameroff S. R. \& Tuszynski J. A. (2002). Quantum computation in brain microtubules: Decoherence and biological feasibility. Phys. Rev. D, 65, 061901.

Hameroff, S. R. \& Penrose, R. (1996). Conscious events as orchestrated space-time selections. Journal of Consciousness Studies, 3 (1), 36-53.

Hughston, L. P. (1996). Geometry of Stochastic State Vector Reduction. Proc. Roy. Soc. Lond. A 452, 953-979.

Pearle, P. (1989). Combining stochastic dynamical state-vector reduction with spontaneous localization. Phys. Rev. A 39, 2277-2289.

Penrose, R. (1996). On gravity's role in quantum state reduction. Gen. Rel. and Grav, 28, 581-600.

Penrose, R. (1994). Shadows of the Mind: a search for the missing science of consciousness. Oxford: Oxford University Press.

Percival, I. C. (1994). Primary state diffusion. Proc. Roy. Soc. Lond. A 447, 189-209.

Puthoff, H. E. \& Targ, R. (1976). A perceptual channel for information transfer over kilometer distances: Historical perspective and recent research. Proc. IEEE, 64, 329-354.

Radin, D. I. \& Nelson, R. D. (1989). Evidence for consciousness-related anomalies in random physical systems. Found. Phys. 19(12), 1499-1514.

Targ, R. \& Puthoff, H.E. (1974). Information transmission under conditions of sensory shielding. Nature 252, $602-$ 607.

Tegmark, M. (2000). Importance of quantum decoherence in brain processes. Phys. Rev. E 61, 4194-4206.

Wackermann, J., Seiter, C., Keibel, H. \& Walach, H. (2003). Correlations between brain electrical activities of two spatially separated human subjects. Neuroscience Letters 336, 60-64.

Address for correspondence: Gao Shan,

The Scientists Work Team of Electro-Magnetic Wave Velocity, Chinese Institute of Electronics, LongZeYuan 24-3-501, Hui Long Guan, ChangPing District, Beijing 102208, P.R. China.

E-mail:rg@mail.ie.ac.cn 